\title{
Refinements of some
}

\section{Hardy-Littlewood-Pólya type inequalities via Green's functions and Fink's identity and related results}

\author{
Sadia Khalid ${ }^{1 *}$ (1) and Josip Pečarić ${ }^{2}$
}

"Correspondence:

saadiakhalid176@gmail.com;

skhalid@cuilahore.edu.pk

'Department of Mathematics, COMSATS University Islamabad Lahore Campus, 54000 Lahore, Pakistan

Full list of author information is available at the end of the article

\begin{abstract}
In this paper, first we present some interesting identities associated with Green's functions and Fink's identity, and further we present some interesting inequalities for $r$-convex functions. We also present refinements of some Hardy-Littlewood-Pólya type inequalities and give an application to the Shannon entropy. Furthermore, we use the Čebyšev functional and Grüss type inequalities and present the bounds for the remainder in the obtained identities. Finally, we use the obtained identities together with Hölder's inequality for integrals and present Ostrowski type inequalities.

MSC: 26A51; 26D15; 52A41

Keywords: Nonincreasing sequence in weighted mean; Shannon entropy; Green's functions; Fink's identity; r-convex function; Čebyšev functional; Grüss type inequality; Ostrowski type inequality; Hölder's inequality
\end{abstract}

\section{Introduction and preliminaries}

The following inequality is given in the well-known book by Hardy, Littlewood, and Pólya [6, Theorem 134]:

Theorem 1.1 If $\vartheta$ is a convex and continuous function defined on $[0, \infty)$ and $a_{i}, i \in \mathbb{N}$, are nonnegative and nonincreasing, then

$$
\vartheta\left(\sum_{i=1}^{m} a_{i}\right)-\vartheta(0)-\sum_{i=1}^{m}\left[\vartheta\left(i a_{i}\right)-\vartheta\left((i-1) a_{i}\right)\right] \geq 0 .
$$

If $\vartheta^{\prime}$ is a strictly increasing function, there is an equality only when $a_{i}$ are equal up to a certain point and then zero.

A weighted case of inequality (1.1) was proved by Bennett [1] for power functions $\vartheta(x)=$ $x^{p}$ in the following way: if $a_{i} \in[0, \infty)$ and $a_{i}(1 \leq i \leq m)$ are nonincreasing and $q_{i} \in[0, \infty)$

(c) The Author(s) 2020. This article is licensed under a Creative Commons Attribution 4.0 International License, which permits use sharing, adaptation, distribution and reproduction in any medium or format, as long as you give appropriate credit to the original author(s) and the source, provide a link to the Creative Commons licence, and indicate if changes were made. The images or other third party material in this article are included in the article's Creative Commons licence, unless indicated otherwise in a credit line to the material. If material is not included in the article's Creative Commons licence and your intended use is not permitted by statutory regulation or exceeds the permitted use, you will need to obtain permission directly from the copyright holder. To view a copy of this licence, visit http://creativecommons.org/licenses/by/4.0/. 
for all $i \in\{1, \ldots, m\}$ such that $Q_{i}=\sum_{k=1}^{i} q_{k}(1 \leq i \leq m)$, then for $p \in(1, \infty)$ the inequality

$$
\left(\sum_{i=1}^{m} q_{i} a_{i}\right)^{p}-\left(q_{1} a_{1}\right)^{p}-\sum_{i=2}^{m} a_{i}^{p}\left[Q_{i}^{p}-Q_{i-1}^{p}\right] \geq 0
$$

holds. If $p \in(0,1)$, then (1.2) holds in the reversed direction (see [1]).

In our work we use nonincreasing $(\searrow$ ) sequence in weighted mean (WM) and nondecreasing $(\nearrow)$ sequence in WM (see [11]) defined as follows.

Definition 1.2 A sequence $\left\{a_{i}\right\}_{i \in \mathbb{N}} \subset \mathbb{R}$ is $\searrow$ in WM if

$$
\frac{1}{Q_{m+1}} \sum_{i=1}^{m+1} q_{i} a_{i} \leq \frac{1}{Q_{m}} \sum_{i=1}^{m} q_{i} a_{i}, \quad m \in \mathbb{N},
$$

where $a_{i}, q_{i} \in \mathbb{R}(i \in \mathbb{N})$ such that $q_{k}>0(1 \leq k \leq i)$ and $Q_{i}:=\sum_{k=1}^{i} q_{k}(i \in \mathbb{N})$.

If (1.3) holds in the reversed direction, then the sequence $\left\{a_{i}\right\}_{i \in \mathbb{N}} \subset \mathbb{R}$ is called $\nearrow$ in WM.

A generalization of inequality (1.2) is presented in [11].

Theorem 1.3 Let $a_{i}, q_{i} \in \mathbb{R}(1 \leq i \leq m)$ such that $a_{i} \geq 0$ and $q_{i}>0$. Let $q_{1} a_{1}, \sum_{i=1}^{m} q_{i} a_{i}$, $Q_{i} a_{i}, Q_{i-1} a_{i} \in[s, t]$ for all $i \in\{2, \ldots, m\}$, and let $\vartheta:[s, t] \rightarrow \mathbb{R}$ be a convex function.

(i) If $\left\{a_{i}\right\}_{i=1}^{m}$ is $\searrow$ in $W M$, then

$$
\vartheta\left(\sum_{i=1}^{m} q_{i} a_{i}\right)-\vartheta\left(q_{1} a_{1}\right)-\sum_{i=2}^{m}\left[\vartheta\left(Q_{i} a_{i}\right)-\vartheta\left(Q_{i-1} a_{i}\right)\right] \geq 0 .
$$

(ii) If $\left\{a_{i}\right\}_{i=1}^{m}$ is $\nearrow$ in $W M$, then

$$
\vartheta\left(\sum_{i=1}^{m} q_{i} a_{i}\right)-\vartheta\left(q_{1} a_{1}\right)-\sum_{i=2}^{m}\left[\vartheta\left(Q_{i} a_{i}\right)-\vartheta\left(Q_{i-1} a_{i}\right)\right] \leq 0 .
$$

If $\vartheta$ is concave, then (1.4) and (1.5) hold in the reversed direction.

Definition 1.4 Let $\mathbf{q}=\left(q_{1}, \ldots, q_{m}\right)$ be a positive probability distribution. Then the Shannon entropy (see [8, 9], and [22]) of $\mathbf{q}$ is defined by $S(\mathbf{q}):=\sum_{i=1}^{m} q_{i} \log \left(\frac{1}{q_{i}}\right)$.

Khalid, Pečarić, and Pečarić presented the following interesting result associated with the Shannon entropy in [8].

Theorem 1.5 Let $q_{i} \in \mathbb{R}$ such that $q_{i}>0(1 \leq i \leq m)$, and let $\vartheta:[s, t] \rightarrow \mathbb{R}$ be a convex function.

(a) Let $0<q_{i}<1(1 \leq i \leq m)$, and let $S(\mathbf{q}), q_{1} \log \left(\frac{1}{q_{1}}\right), Q_{i} \log \left(\frac{1}{q_{i}}\right), Q_{i-1} \log \left(\frac{1}{q_{i}}\right) \in[s, t]$ for all $i \in\{2, \ldots, m\}$.

(i) If $\left\{q_{i}\right\}_{i=1}^{m}$ is $\searrow$, then

$$
\begin{aligned}
& \vartheta(S(\mathbf{q}))-\vartheta\left(q_{1} \log \left(\frac{1}{q_{1}}\right)\right) \\
& -\sum_{i=2}^{m}\left[\vartheta\left(Q_{i} \log \left(\frac{1}{q_{i}}\right)\right)-\vartheta\left(Q_{i-1} \log \left(\frac{1}{q_{i}}\right)\right)\right] \leq 0 .
\end{aligned}
$$


(ii) If $\left\{q_{i}\right\}_{i=1}^{m}$ is $\nearrow$, then

$$
\begin{aligned}
& \vartheta(S(\mathbf{q}))-\vartheta\left(q_{1} \log \left(\frac{1}{q_{1}}\right)\right) \\
& \quad-\sum_{i=2}^{m}\left[\vartheta\left(Q_{i} \log \left(\frac{1}{q_{i}}\right)\right)-\vartheta\left(Q_{i-1} \log \left(\frac{1}{q_{i}}\right)\right)\right] \geq 0 .
\end{aligned}
$$

(b) Let $q_{i} \geq 1(1 \leq i \leq m)$ and let $-S(\mathbf{q}), q_{1} \log q_{1}, Q_{i} \log q_{i}, Q_{i-1} \log q_{i} \in[s, t]$ for all $i \in\{2, \ldots, m\}$.

(i) If $\left\{q_{i}\right\}_{i=1}^{m}$ is $\searrow$, then

$$
\vartheta(-S(\mathbf{q}))-\vartheta\left(q_{1} \log q_{1}\right)-\sum_{i=2}^{m}\left[\vartheta\left(Q_{i} \log q_{i}\right)-\vartheta\left(Q_{i-1} \log q_{i}\right)\right] \geq 0
$$

(ii) If $\left\{q_{i}\right\}_{i=1}^{m}$ is $\nearrow$, then

$$
\vartheta(-S(\mathbf{q}))-\vartheta\left(q_{1} \log q_{1}\right)-\sum_{i=2}^{m}\left[\vartheta\left(Q_{i} \log q_{i}\right)-\vartheta\left(Q_{i-1} \log q_{i}\right)\right] \leq 0
$$

If $\vartheta$ is concave, then (1.6)-(1.9) hold in the reversed direction.

In the second section, we generalize inequalities (1.4), (1.7), and (1.8) for $r$-convex functions, and we also present refinements of these inequalities.

Consider the Green's functions $G_{\delta}:[s, t] \times[s, t] \rightarrow \mathbb{R}(\delta \in\{1, \ldots, 5\})$ defined as follows (see $[4,7,13,14]$, and [16-19]):

$$
\begin{aligned}
& G_{1}(x, \tilde{u})= \begin{cases}\frac{(x-t)(\tilde{u}-s)}{t-s}, & s \leq \tilde{u} \leq x, \\
\frac{(\tilde{u}-t)(x-s)}{t-s}, & x \leq \tilde{u} \leq t,\end{cases} \\
& G_{2}(x, \tilde{u})= \begin{cases}s-\tilde{u}, & s \leq \tilde{u} \leq x, \\
s-x, & x<\tilde{u} \leq t,\end{cases} \\
& G_{3}(x, \tilde{u})= \begin{cases}x-t, & s \leq \tilde{u} \leq x, \\
\tilde{u}-t, & x<\tilde{u} \leq t,\end{cases} \\
& G_{4}(x, \tilde{u})= \begin{cases}x-s, & s \leq \tilde{u} \leq x, \\
\tilde{u}-s, & x<\tilde{u} \leq t,\end{cases}
\end{aligned}
$$

and

$$
G_{5}(x, \tilde{u})= \begin{cases}t-\tilde{u}, & s \leq \tilde{u} \leq x \\ t-x, & x<\tilde{u} \leq t\end{cases}
$$

\section{Remark 1.6}

(i) It is easy to see that the functions $G_{\delta}(x, \tilde{u})(\delta \in\{1, \ldots, 5\})$ defined in (1.10)-(1.14) are continuous, symmetric, and convex with respect to both the variables $x$ and $\tilde{u}$.

(ii) Throughout this paper, for $G_{\delta}$, we consider $\delta \in\{1, \ldots, 5\}$. 
For $G_{\delta}(x, \tilde{u})$ defined in (1.10)-(1.14) and for a twice differentiable function $\vartheta$ defined on $[s, t]$, we can consider the following expression:

$$
\int_{s}^{t} G_{\delta}(x, \tilde{u}) \vartheta^{\prime \prime}(\tilde{u}) d \tilde{u}=\int_{s}^{x} G_{\delta}(x, \tilde{u}) \vartheta^{\prime \prime}(\tilde{u}) d \tilde{u}+\int_{x}^{t} G_{\delta}(x, \tilde{u}) \vartheta^{\prime \prime}(\tilde{u}) d \tilde{u} .
$$

Now if we use the values of $G_{\delta}(x, \tilde{u})$ over the intervals $[s, x]$ and $[x, t]$, then the following result is valid (see [4] and [13]).

Lemma 1.7 Let $\vartheta:[s, t] \rightarrow \mathbb{R}$ be a function such that $\vartheta \in C^{2}([s, t])$, and let $G_{\delta}$ be the Green's functions defined in (1.10)-(1.14). Then

$$
\begin{aligned}
& \vartheta(x)=\frac{t-x}{t-s} \vartheta(s)+\frac{x-s}{t-s} \vartheta(t)+\int_{s}^{t} G_{1}(x, \tilde{u}) \vartheta^{\prime \prime}(\tilde{u}) d \tilde{u}, \\
& \vartheta(x)=\vartheta(s)+(x-s) \vartheta^{\prime}(t)+\int_{s}^{t} G_{2}(x, \tilde{u}) \vartheta^{\prime \prime}(\tilde{u}) d \tilde{u}, \\
& \vartheta(x)=\vartheta(t)-(t-x) \vartheta^{\prime}(s)+\int_{s}^{t} G_{3}(x, \tilde{u}) \vartheta^{\prime \prime}(\tilde{u}) d \tilde{u}, \\
& \vartheta(x)=\vartheta(t)-(t-s) \vartheta^{\prime}(t)+(x-s) \vartheta^{\prime}(s)+\int_{s}^{t} G_{4}(x, \tilde{u}) \vartheta^{\prime \prime}(\tilde{u}) d \tilde{u},
\end{aligned}
$$

and

$$
\vartheta(x)=\vartheta(s)+(t-s) \vartheta^{\prime}(s)-(t-x) \vartheta^{\prime}(t)+\int_{s}^{t} G_{5}(x, \tilde{u}) \vartheta^{\prime \prime}(\tilde{u}) d \tilde{u} .
$$

In the second section, we prove some interesting identities and inequalities for $r$-convex functions by using Lemma 1.7 and the following Fink's identity (see [5, 10], and [12]).

Theorem 1.8 Let $s, t \in \mathbb{R}, \vartheta:[s, t] \rightarrow \mathbb{R}, r \geq 1$ and $\vartheta^{(r-1)}$ be absolutely continuous on $[s, t]$. Then

$$
\begin{aligned}
\vartheta(x)= & \frac{r}{t-s} \int_{s}^{t} \vartheta(u) d u+\frac{1}{(r-1) !(t-s)} \int_{s}^{t}(x-u)^{r-1} k(u, x) \vartheta^{(r)}(u) d u \\
& -\frac{1}{t-s} \sum_{n=1}^{r-1} \frac{r-n}{n !}\left[(x-s)^{n} \vartheta^{(n-1)}(s)-(x-t)^{n} \vartheta^{(n-1)}(t)\right],
\end{aligned}
$$

where

$$
k(u, x)= \begin{cases}u-s, & s \leq u \leq x \leq t \\ u-t, & s \leq x<u \leq t\end{cases}
$$

Let $\vartheta$ be a real-valued function defined on $[s, t]$. A criterion to check the $r$-convexity $(r \geq 0)$ of a function $\vartheta$ is the following:

Theorem 1.9 If $\vartheta^{(r)}$ exists, then $\vartheta$ is $r$-convex if and only if $\vartheta^{(r)} \geq 0$. 
In the third section, we present some interesting results by using the following Čebyšev functional and Grüss type inequalities (see [2] and [3]):

Let $L_{p}[s, t](1 \leq p<\infty)$ and $L_{\infty}[s, t]$ denote the space of p-power integrable functions and the space of essentially bounded functions defined on $[s, t]$ respectively together with the norms

$$
\|\zeta\|_{p}=\left(\int_{s}^{t}|\zeta(u)|^{p} d u\right)^{\frac{1}{p}} \text { and }\|\zeta\|_{\infty}=\operatorname{ess} \sup _{u \in[s, t]}|\zeta(u)|
$$

respectively.

Suppose that $\zeta_{1}, \zeta_{2}:[s, t] \rightarrow \mathbb{R}$ are two Lebesgue integrable functions. The Čebyšev functional is defined by

$$
\Lambda\left(\zeta_{1}, \zeta_{2}\right):=\frac{1}{t-s} \int_{s}^{t} \zeta_{1}(u) \zeta_{2}(u) d u-\frac{1}{t-s} \int_{s}^{t} \zeta_{1}(u) d u \cdot \frac{1}{t-s} \int_{s}^{t} \zeta_{2}(u) d u .
$$

Cerone and Dragomir proved the next two results related to Grüss type inequalities in [2].

Theorem 1.10 Suppose that $\zeta_{1}:[s, t] \rightarrow \mathbb{R}$ is a Lebesgue integrable function and $\zeta_{2}:$ $[s, t] \rightarrow \mathbb{R}$ is an absolutely continuous function such that $(\cdot-s)(t-\cdot)\left(\zeta_{2}^{\prime}\right)^{2} \in L_{1}[s, t]$. Then

$$
\left|\Lambda\left(\zeta_{1}, \zeta_{2}\right)\right| \leq \sqrt{\frac{\Lambda\left(\zeta_{1}, \zeta_{1}\right)}{2(t-s)}} \cdot \sqrt{\int_{s}^{t}(u-s)(t-u)\left(\zeta_{2}^{\prime}(u)\right)^{2} d u} .
$$

Theorem 1.11 Suppose that $\zeta_{1}:[s, t] \rightarrow \mathbb{R}$ is absolutely continuous such that $\zeta_{1}^{\prime} \in L_{\infty}[s, t]$ and $\zeta_{2}:[s, t] \rightarrow \mathbb{R}$ is monotonically $\nearrow$. Then

$$
\left|\Lambda\left(\zeta_{1}, \zeta_{2}\right)\right| \leq \frac{1}{2(t-s)}\left\|\zeta_{1}^{\prime}\right\|_{\infty} \int_{s}^{t}(u-s)(t-u) d \zeta_{2}(u)
$$

The constants $\frac{1}{\sqrt{2}}$ and $\frac{1}{2}$ are the best possible in (1.23) and (1.24) respectively.

The organization of this paper is as follows: in the second section, we obtain some interesting identities related to Green's functions and Fink's result. Further, we use these identities and generalize inequalities of kind (1.4), (1.7), and (1.8) for $r$-convex functions. In addition, we also present refinements of these inequalities and give an application to the Shannon entropy. In the third section, we use the Čebyšev functional and Grüss type inequalities and find the new bounds for the remainder in the obtained identities. In the fourth section, we use the identities from section two together with Hölder's inequality for integrals and obtain Ostrowski type inequalities (see [20] and [21]).

\section{Refinements of some Hardy-Littlewood-Pólya type inequalities and an application to the Shannon entropy}

The first main theorem is related to the following identity which will play an important role in our paper.

Theorem 2.1 Let $\vartheta:[s, t] \rightarrow \mathbb{R}$ and $\vartheta^{(r-1)}$ be absolutely continuous for $r \geq 3$. Let $a_{i}, q_{i} \in \mathbb{R}$ $(1 \leq i \leq m)$, and let $q_{1} a_{1}, \sum_{i=1}^{m} q_{i} a_{i}, Q_{i} a_{i}, Q_{i-1} a_{i} \in[s, t]$ for all $i \in\{2, \ldots, m\}$. Let $G_{\delta}(x, \tilde{u})$ 
and $k(u, x)$ be defined in (1.10)-(1.14) and (1.21) respectively. Then

$$
\begin{aligned}
& \vartheta\left(\sum_{i=1}^{m} q_{i} a_{i}\right)-\vartheta\left(q_{1} a_{1}\right)-\sum_{i=2}^{m}\left[\vartheta\left(Q_{i} a_{i}\right)-\vartheta\left(Q_{i-1} a_{i}\right)\right] \\
& =\frac{1}{t-s} \sum_{n=0}^{r-3} \frac{r-n-2}{n !} \int_{s}^{t}\left[G_{\delta}\left(\sum_{i=1}^{m} q_{i} a_{i}, \tilde{u}\right)-G_{\delta}\left(q_{1} a_{1}, \tilde{u}\right)\right. \\
& \left.\quad-\sum_{i=2}^{m}\left[G_{\delta}\left(Q_{i} a_{i}, \tilde{u}\right)-G_{\delta}\left(Q_{i-1} a_{i}, \tilde{u}\right)\right]\right] \\
& \quad \times\left[(\tilde{u}-t)^{n} \vartheta^{(n+1)}(t)-(\tilde{u}-s)^{n} \vartheta^{(n+1)}(s)\right] d \tilde{u} \\
& \quad+\frac{1}{(t-s)(r-3) !} \int_{s}^{t} \vartheta^{(r)}(u)\left[\int _ { s } ^ { t } \left[G_{\delta}\left(\sum_{i=1}^{m} q_{i} a_{i}, \tilde{u}\right)-G_{\delta}\left(q_{1} a_{1}, \tilde{u}\right)\right.\right. \\
& \left.\left.\quad-\sum_{i=2}^{m}\left[G_{\delta}\left(Q_{i} a_{i}, \tilde{u}\right)-G_{\delta}\left(Q_{i-1} a_{i}, \tilde{u}\right)\right]\right](\tilde{u}-u)^{r-3} k(u, \tilde{u}) d \tilde{u}\right] d u .
\end{aligned}
$$

Proof Let $\delta=1$. Using (1.15) in the L.H.S of (1.4), we have

$$
\begin{gathered}
\vartheta\left(\sum_{i=1}^{m} q_{i} a_{i}\right)-\vartheta\left(q_{1} a_{1}\right)-\sum_{i=2}^{m}\left[\vartheta\left(Q_{i} a_{i}\right)-\vartheta\left(Q_{i-1} a_{i}\right)\right] \\
=\frac{\vartheta(t)-\vartheta(s)}{t-s}\left[\sum_{i=1}^{m} q_{i} a_{i}-q_{1} a_{1}-\sum_{i=2}^{m}\left[Q_{i} a_{i}-Q_{i-1} a_{i}\right]\right] \\
+\int_{s}^{t}\left[G_{1}\left(\sum_{i=1}^{m} q_{i} a_{i}, \tilde{u}\right)-G_{1}\left(q_{1} a_{1}, \tilde{u}\right)\right. \\
\quad-\sum_{i=2}^{m}\left[G_{1}\left(Q_{i} a_{i}, \tilde{u}\right)-G_{1}\left(Q_{i-1} a_{i}, \tilde{u}\right)\right] \vartheta^{\prime \prime}(\tilde{u}) d \tilde{u}
\end{gathered}
$$

equivalent to

$$
\begin{aligned}
\vartheta\left(\sum_{i=1}^{m} q_{i} a_{i}\right)-\vartheta\left(q_{1} a_{1}\right)-\sum_{i=2}^{m}\left[\vartheta\left(Q_{i} a_{i}\right)-\vartheta\left(Q_{i-1} a_{i}\right)\right] \\
=\int_{s}^{t}\left[G_{1}\left(\sum_{i=1}^{m} q_{i} a_{i}, \tilde{u}\right)-G_{1}\left(q_{1} a_{1}, \tilde{u}\right)\right. \\
\left.\quad-\sum_{i=2}^{m}\left[G_{1}\left(Q_{i} a_{i}, \tilde{u}\right)-G_{1}\left(Q_{i-1} a_{i}, \tilde{u}\right)\right]\right] \vartheta^{\prime \prime}(\tilde{u}) d \tilde{u} .
\end{aligned}
$$

By taking Fink's identity (1.20), it is obvious that

$$
\begin{aligned}
\vartheta^{\prime \prime}(x)= & \frac{1}{t-s} \sum_{n=0}^{r-3} \frac{r-n-2}{n !}\left[(x-t)^{n} \vartheta^{(n+1)}(t)-(x-s)^{n} \vartheta^{(n+1)}(s)\right] \\
& +\frac{1}{(t-s)(r-3) !} \int_{s}^{t}(x-u)^{r-3} k(u, x) \vartheta^{(r)}(u) d u .
\end{aligned}
$$


Using (2.3) in (2.2), we have

$$
\begin{aligned}
\vartheta\left(\sum_{i=1}^{m} q_{i} a_{i}\right)-\vartheta\left(q_{1} a_{1}\right)-\sum_{i=2}^{m}\left[\vartheta\left(Q_{i} a_{i}\right)-\vartheta\left(Q_{i-1} a_{i}\right)\right] \\
=\frac{1}{t-s} \int_{s}^{t}\left[G_{1}\left(\sum_{i=1}^{m} q_{i} a_{i}, \tilde{u}\right)-G_{1}\left(q_{1} a_{1}, \tilde{u}\right)\right. \\
\left.\quad-\sum_{i=2}^{m}\left[G_{1}\left(Q_{i} a_{i}, \tilde{u}\right)-G_{1}\left(Q_{i-1} a_{i}, \tilde{u}\right)\right]\right] \\
\quad \times \sum_{n=0}^{r-3} \frac{r-n-2}{n !}\left[(\tilde{u}-t)^{n} \vartheta^{(n+1)}(t)-(\tilde{u}-s)^{n} \vartheta^{(n+1)}(s)\right] d \tilde{u} \\
\quad+\frac{1}{(t-s)(r-3) !} \int_{s}^{t}\left[G_{1}\left(\sum_{i=1}^{m} q_{i} a_{i}, \tilde{u}\right)-G_{1}\left(q_{1} a_{1}, \tilde{u}\right)\right. \\
\quad-\sum_{i=2}^{m}\left[G_{1}\left(Q_{i} a_{i}, \tilde{u}\right)-G_{1}\left(Q_{i-1} a_{i}, \tilde{u}\right)\right] \\
\quad \times\left[\int_{s}^{t}(\tilde{u}-u)^{r-3} k(u, \tilde{u}) \vartheta^{(r)}(u) d u\right] d \tilde{u} .
\end{aligned}
$$

Now interchange the integral and summation in the first term and apply Fubini's theorem in the second term, identity (2.1) is immediate for $\delta=1$.

Furthermore, using identities (1.16)-(1.19) in the LHS of inequality (1.4) and following the proof as given for the case $\delta=1$, we obtain identity $(2.1)$ for $\delta \in\{2, \ldots, 5\}$.

We present inequality (1.4) for $r$-convex functions as follows.

Theorem 2.2 Let all the assumptions of Theorem 2.1 be satisfied, and let for $r \geq 3, \vartheta$ be r-convex. If

$$
\begin{aligned}
\int_{s}^{t} & {\left[G_{\delta}\left(\sum_{i=1}^{m} q_{i} a_{i}, \tilde{u}\right)-G_{\delta}\left(q_{1} a_{1}, \tilde{u}\right)-\sum_{i=2}^{m}\left[G_{\delta}\left(Q_{i} a_{i}, \tilde{u}\right)-G_{\delta}\left(Q_{i-1} a_{i}, \tilde{u}\right)\right]\right] } \\
& \times(\tilde{u}-u)^{r-3} k(u, \tilde{u}) d \tilde{u} \geq 0
\end{aligned}
$$

holds, then

$$
\begin{aligned}
& \vartheta\left(\sum_{i=1}^{m} q_{i} a_{i}\right)-\vartheta\left(q_{1} a_{1}\right)-\sum_{i=2}^{m}\left[\vartheta\left(Q_{i} a_{i}\right)-\vartheta\left(Q_{i-1} a_{i}\right)\right] \\
& \geq \frac{1}{t-s} \sum_{n=0}^{r-3} \frac{r-n-2}{n !} \int_{s}^{t}\left[G_{\delta}\left(\sum_{i=1}^{m} q_{i} a_{i}, \tilde{u}\right)-G_{\delta}\left(q_{1} a_{1}, \tilde{u}\right)\right. \\
& \left.\quad-\sum_{i=2}^{m}\left[G_{\delta}\left(Q_{i} a_{i}, \tilde{u}\right)-G_{\delta}\left(Q_{i-1} a_{i}, \tilde{u}\right)\right]\right] \\
& \quad \times\left[(\tilde{u}-t)^{n} \vartheta^{(n+1)}(t)-(\tilde{u}-s)^{n} \vartheta^{(n+1)}(s)\right] d \tilde{u} .
\end{aligned}
$$

If the reversed inequality holds in (2.4), then (2.5) holds in the reversed direction. 
Proof The absolute continuity of $\vartheta^{(r-1)}$ defined on $[s, t]$ implies the existence of $\vartheta^{(r)}$ almost everywhere. We use the $r$-convexity of $\vartheta$, and from Theorem 1.9 we have $\vartheta^{(r)}(x) \geq 0$ for all $x \in[s, t]$. Use the nonnegativity of $\vartheta^{(r)}$ together with inequality (2.4) in (2.1), inequality (2.5) is immediate.

Theorem 2.3 Let $\vartheta:[s, t] \rightarrow \mathbb{R}$ be continuous and convex, and let $G_{\delta}$ be the Green's functions defined in (1.10)-(1.14). Let $a_{i}, q_{i} \in \mathbb{R}(1 \leq i \leq m)$, and let $q_{1} a_{1}, \sum_{i=1}^{m} q_{i} a_{i}, Q_{i} a_{i}$, $Q_{i-1} a_{i} \in[s, t]$ for all $i \in\{2, \ldots, m\}$. Then the following statements are equivalent:

(i) For every continuous convex function $\vartheta$, inequality (1.4) holds.

(ii) For the Green's functions $G_{\delta}$ and for all $\tilde{u} \in[s, t]$, we have

$$
G_{\delta}\left(\sum_{i=1}^{m} q_{i} a_{i}, \tilde{u}\right)-G_{\delta}\left(q_{1} a_{1}, \tilde{u}\right)-\sum_{i=2}^{m}\left[G_{\delta}\left(Q_{i} a_{i}, \tilde{u}\right)-G_{\delta}\left(Q_{i-1} a_{i}, \tilde{u}\right)\right] \geq 0 .
$$

If the reversed inequalities hold in (1.4) and (2.6), then statements (i) and (ii) are also equivalent.

Proof The idea of the proof is the same as given in [15].

Let statement (i) be satisfied. As the functions $G_{\delta}(\cdot, \tilde{u})$ are continuous and convex for all $\delta \in\{1, \ldots, 5\}$ such that $\tilde{u} \in[s, t]$, inequality (1.4) also holds for these functions $G_{\delta}(\cdot, \tilde{u})$ for every fixed $\delta$ and inequality (2.6) is immediate.

Let statement (ii) be satisfied, and let $\vartheta$ be a twice differentiable convex function. As from Lemma 1.7 the function $\vartheta$ can be represented in the forms (1.15)-(1.19), it is easy to see that

$$
\begin{aligned}
& \vartheta\left(\sum_{i=1}^{m} q_{i} a_{i}\right)-\vartheta\left(q_{1} a_{1}\right)-\sum_{i=2}^{m}\left[\vartheta\left(Q_{i} a_{i}\right)-\vartheta\left(Q_{i-1} a_{i}\right)\right] \\
& =\int_{s}^{t}\left[G_{\delta}\left(\sum_{i=1}^{m} q_{i} a_{i}, \tilde{u}\right)-G_{\delta}\left(q_{1} a_{1}, \tilde{u}\right)-\sum_{i=2}^{m}\left[G_{\delta}\left(Q_{i} a_{i}, \tilde{u}\right)-G_{\delta}\left(Q_{i-1} a_{i}, \tilde{u}\right)\right]\right] \\
& \quad \times \vartheta^{\prime \prime}(\tilde{u}) d \tilde{u} .
\end{aligned}
$$

Now use inequality (2.6) together with $\vartheta^{\prime \prime}(\tilde{u}) \geq 0$ for all $\tilde{u} \in[s, t]$ in (2.7), inequality (1.4) is immediate.

The differentiability condition can be eliminated here as it is possible to approximate uniformly a continuous convex function by convex polynomials (see [23, p. 172]).

We present refinement of inequality (1.4) as follows.

Theorem 2.4 Let all the assumptions of Theorem 2.1 be satisfied, and let $r$ be even such that $r>3$. Let $a_{i}, q_{i} \in \mathbb{R}(1 \leq i \leq m)$ such that $a_{i} \geq 0$ and $q_{i}>0$, and let the sequence $\left\{a_{i}\right\}_{i=1}^{m}$ be $\searrow$ in WM.

(i) If $\vartheta:[s, t] \rightarrow \mathbb{R}$ is r-convex, then (2.5) holds. 
(ii) Let (2.5) be satisfied, and let $\Gamma:[s, t] \rightarrow \mathbb{R}$ be a function defined by

$$
\begin{aligned}
\Gamma(x)= & \frac{1}{t-s} \int_{s}^{t} G_{\delta}(x, \tilde{u}) \sum_{n=0}^{r-3} \frac{r-n-2}{n !} \\
& \times\left[(\tilde{u}-t)^{n} \vartheta^{(n+1)}(t)-(\tilde{u}-s)^{n} \vartheta^{(n+1)}(s)\right] d \tilde{u} .
\end{aligned}
$$

If $\Gamma$ is convex, then the RHS of (2.5) is nonnegative and we have

$$
\vartheta\left(\sum_{i=1}^{m} q_{i} a_{i}\right)-\vartheta\left(q_{1} a_{1}\right) \geq \sum_{i=2}^{m}\left[\vartheta\left(Q_{i} a_{i}\right)-\vartheta\left(Q_{i-1} a_{i}\right)\right]
$$

Proof Using the convex functions $G_{\delta}$ in (1.4), we have

$$
G_{\delta}\left(\sum_{i=1}^{m} q_{i} a_{i}, \tilde{u}\right)-G_{\delta}\left(q_{1} a_{1}, \tilde{u}\right)-\sum_{i=2}^{m}\left[G_{\delta}\left(Q_{i} a_{i}, \tilde{u}\right)-G_{\delta}\left(Q_{i-1} a_{i}, \tilde{u}\right)\right] \geq 0 .
$$

For $s \leq \tilde{u} \leq u$, the inequalities

$$
\begin{aligned}
& \int_{s}^{u}\left[G_{\delta}\left(\sum_{i=1}^{m} q_{i} a_{i}, \tilde{u}\right)-G_{\delta}\left(q_{1} a_{1}, \tilde{u}\right)-\sum_{i=2}^{m}\left[G_{\delta}\left(Q_{i} a_{i}, \tilde{u}\right)-G_{\delta}\left(Q_{i-1} a_{i}, \tilde{u}\right)\right]\right] \\
& \quad \times(\tilde{u}-u)^{r-3} k(u, \tilde{u}) d \tilde{u} \geq 0
\end{aligned}
$$

and

$$
\begin{aligned}
& \int_{s}^{u}\left[G_{\delta}\left(\sum_{i=1}^{m} q_{i} a_{i}, \tilde{u}\right)-G_{\delta}\left(q_{1} a_{1}, \tilde{u}\right)-\sum_{i=2}^{m}\left[G_{\delta}\left(Q_{i} a_{i}, \tilde{u}\right)-G_{\delta}\left(Q_{i-1} a_{i}, \tilde{u}\right)\right]\right] \\
& \quad \times(\tilde{u}-u)^{r-3} k(u, \tilde{u}) d \tilde{u} \leq 0
\end{aligned}
$$

hold for even $r$ such that $r>3$ and for odd $r$ such that $r \geq 3$ respectively. For $u \leq \tilde{u} \leq t$, the inequality

$$
\begin{aligned}
& \int_{u}^{t}\left[G_{\delta}\left(\sum_{i=1}^{m} q_{i} a_{i}, \tilde{u}\right)-G_{\delta}\left(q_{1} a_{1}, \tilde{u}\right)-\sum_{i=2}^{m}\left[G_{\delta}\left(Q_{i} a_{i}, \tilde{u}\right)-G_{\delta}\left(Q_{i-1} a_{i}, \tilde{u}\right)\right]\right] \\
& \quad \times(\tilde{u}-u)^{r-3} k(u, \tilde{u}) d \tilde{u} \geq 0
\end{aligned}
$$

holds for $r \geq 3$.

(i) Inequality (2.9) together with inequality (2.10) yields inequality (2.4) for even $r$ such that $r>3$. As $\vartheta$ is $r$-convex for even $r$ such that $r>3$, applying Theorem 2.2, we obtain (2.5).

(ii) Clearly, inequality (2.5) can be written as

$$
\begin{aligned}
& \vartheta\left(\sum_{i=1}^{m} q_{i} a_{i}\right)-\vartheta\left(q_{1} a_{1}\right)-\sum_{i=2}^{m}\left[\vartheta\left(Q_{i} a_{i}\right)-\vartheta\left(Q_{i-1} a_{i}\right)\right] \\
& \quad \geq \Gamma\left(\sum_{i=1}^{m} q_{i} a_{i}\right)-\Gamma\left(q_{1} a_{1}\right)-\sum_{i=2}^{m}\left[\Gamma\left(Q_{i} a_{i}\right)-\Gamma\left(Q_{i-1} a_{i}\right)\right] .
\end{aligned}
$$


As the sequence $\left\{a_{i}\right\}_{i=1}^{m}$ is $\searrow$ in WM, replace $\vartheta$ by $\Gamma$ in Theorem 1.3 (i), the nonnegativity of the RHS of (2.11) is immediate, and we obtain (2.8).

An application to the Shannon entropy is the following:

Corollary 2.5 Let all the assumptions of Theorem 2.1 be satisfied, and let $\mathbf{q}=\left(q_{1}, \ldots, q_{m}\right)$ be a positive probability distribution. Let $r$ be even such that $r>3$, and let $\vartheta:[s, t] \rightarrow \mathbb{R}$ be $r$-convex.

(i) Let $S(\mathbf{q}), q_{1} \log \left(\frac{1}{q_{1}}\right), Q_{i} \log \left(\frac{1}{q_{i}}\right), Q_{i-1} \log \left(\frac{1}{q_{i}}\right) \in[s, t]$ for all $i \in\{2, \ldots, m\}$, where $0<q_{i}<1(1 \leq i \leq m)$. Then

$$
\begin{aligned}
\vartheta(S(\mathbf{q})) & -\vartheta\left(q_{1} \log \left(\frac{1}{q_{1}}\right)\right) \\
& -\sum_{i=2}^{m}\left[\vartheta\left(Q_{i} \log \left(\frac{1}{q_{i}}\right)\right)-\vartheta\left(Q_{i-1} \log \left(\frac{1}{q_{i}}\right)\right)\right] \\
\geq & \frac{1}{t-s} \sum_{n=0}^{r-3} \frac{r-n-2}{n !} \int_{s}^{t}\left[G_{\delta}(S(\mathbf{q}), \tilde{u})-G_{\delta}\left(q_{1} \log \left(\frac{1}{q_{1}}\right), \tilde{u}\right)\right. \\
& \left.-\sum_{i=2}^{m}\left[G_{\delta}\left(Q_{i} \log \left(\frac{1}{q_{i}}\right), \tilde{u}\right)-G_{\delta}\left(Q_{i-1} \log \left(\frac{1}{q_{i}}\right), \tilde{u}\right)\right]\right] \\
& \times\left[(\tilde{u}-t)^{n} \vartheta^{(n+1)}(t)-(\tilde{u}-s)^{n} \vartheta^{(n+1)}(s)\right] d \tilde{u} .
\end{aligned}
$$

(ii) Let $-S(\mathbf{q}), q_{1} \log q_{1}, Q_{i} \log q_{i}, Q_{i-1} \log q_{i} \in[s, t]$ for all $i \in\{2, \ldots, m\}$, where $q_{i} \geq 1$ $(1 \leq i \leq m)$. Then

$$
\begin{aligned}
& \vartheta(-S(\mathbf{q}))-\vartheta\left(q_{1} \log q_{1}\right)-\sum_{i=2}^{m}\left[\vartheta\left(Q_{i} \log q_{i}\right)-\vartheta\left(Q_{i-1} \log q_{i}\right)\right] \\
& \geq \frac{1}{t-s} \sum_{n=0}^{r-3} \frac{r-n-2}{n !} \int_{s}^{t}\left[G_{\delta}(-S(\mathbf{q}), \tilde{u})-G_{\delta}\left(q_{1} \log q_{1}, \tilde{u}\right)\right. \\
& \left.\quad-\sum_{i=2}^{m}\left[G_{\delta}\left(Q_{i} \log q_{i}, \tilde{u}\right)-G_{\delta}\left(Q_{i-1} \log q_{i}, \tilde{u}\right)\right]\right] \\
& \quad \times\left[(\tilde{u}-t)^{n} \vartheta^{(n+1)}(t)-(\tilde{u}-s)^{n} \vartheta^{(n+1)}(s)\right] d \tilde{u} .
\end{aligned}
$$

\section{Proof}

(i) Take $a_{i}=\log \left(\frac{1}{q_{i}}\right)$ and use Theorem 2.4(i), (2.12) is immediate.

(ii) Taking $a_{i}=-\log \left(\frac{1}{q_{i}}\right)$ and following the proof of (i), we obtain (2.13).

Remark 2.6 Special cases when $\sum_{i=1}^{m} q_{i} a_{i}=\sum_{i=1}^{m} q_{i} \log \left(\frac{1}{q_{i}}\right)=S(\mathbf{q}), 0<q_{i}<1$, and $\sum_{i=1}^{m} q_{i} a_{i}=-\sum_{i=1}^{m} q_{i} \log \left(\frac{1}{q_{i}}\right)=-S(\mathbf{q}), q_{i} \geq 1$, also hold. 


\section{Grüss type inequality-upper bounds for the remainder in the obtained identity}

In this section we present some new bounds for the remainder in the obtained identity. We denote

$$
\begin{aligned}
\varphi(u)= & \int_{s}^{t}\left[G_{\delta}\left(\sum_{i=1}^{m} q_{i} a_{i}, \tilde{u}\right)-G_{\delta}\left(q_{1} a_{1}, \tilde{u}\right)-\sum_{i=2}^{m} G_{\delta}\left(Q_{i} a_{i}, \tilde{u}\right)\right. \\
& \left.+\sum_{i=2}^{m} G_{\delta}\left(Q_{i-1} a_{i}, \tilde{u}\right)\right](\tilde{u}-u)^{r-3} k(u, \tilde{u}) d \tilde{u},
\end{aligned}
$$

where $q_{1} a_{1}, \sum_{i=1}^{m} q_{i} a_{i}, Q_{i} a_{i}, Q_{i-1} a_{i} \in[s, t]$ for all $i \in\{2, \ldots, m\}$. In addition $G_{\delta}(\cdot, \tilde{u})$ and $k(u, \tilde{u})$, appearing in (3.1), are defined in (1.10)-(1.14) and (1.21) respectively.

Theorem 3.1 Let $\vartheta:[s, t] \rightarrow \mathbb{R}, \vartheta^{(r)}$ be absolutely continuous for $r \geq 3$ with $(\cdot-s)(t-\cdot)$ $\left(\vartheta^{(r+1)}\right)^{2} \in L_{1}[s, t]$, and let $G_{\delta}$ and $\Lambda$ be the same as defined in (1.10)-(1.14) and (1.22) respectively. Let $q_{1} a_{1}, \sum_{i=1}^{m} q_{i} a_{i}, Q_{i} a_{i}, Q_{i-1} a_{i} \in[s, t]$ for all $i \in\{2, \ldots, m\}$. If $\varphi$ is defined in (3.1), then

$$
\begin{aligned}
& \vartheta\left(\sum_{i=1}^{m} q_{i} a_{i}\right)-\vartheta\left(q_{1} a_{1}\right)-\sum_{i=2}^{m}\left[\vartheta\left(Q_{i} a_{i}\right)-\vartheta\left(Q_{i-1} a_{i}\right)\right] \\
& =\frac{1}{t-s} \sum_{n=0}^{r-3} \frac{r-n-2}{n !} \int_{s}^{t}\left[G_{\delta}\left(\sum_{i=1}^{m} q_{i} a_{i}, \tilde{u}\right)-G_{\delta}\left(q_{1} a_{1}, \tilde{u}\right)\right. \\
& \left.\quad-\sum_{i=2}^{m}\left[G_{\delta}\left(Q_{i} a_{i}, \tilde{u}\right)-G_{\delta}\left(Q_{i-1} a_{i}, \tilde{u}\right)\right]\right] \\
& \quad \times\left[(\tilde{u}-t)^{n} \vartheta^{(n+1)}(t)-(\tilde{u}-s)^{n} \vartheta^{(n+1)}(s)\right] d \tilde{u} \\
& \quad+\frac{\vartheta^{(r-1)}(t)-\vartheta^{(r-1)}(s)}{(t-s)^{2}(r-3) !} \int_{s}^{t} \varphi(u) d u+\Xi_{r}(s, t ; \vartheta),
\end{aligned}
$$

where the remainder $\Xi_{r}(s, t ; \vartheta)$ satisfies the estimation

$$
\begin{aligned}
\left|\Xi_{r}(s, t ; \vartheta)\right| \leq & \frac{1}{(r-3) !} \cdot \sqrt{\frac{\Lambda(\varphi(u), \varphi(u))}{2(t-s)}} \\
& \times \sqrt{\int_{s}^{t}(u-s)(t-u)\left(\vartheta^{(r+1)}(u)\right)^{2} d u} .
\end{aligned}
$$

Proof Applying Theorem 1.10 for $\zeta_{1} \rightarrow \varphi$ and $\zeta_{2} \rightarrow \vartheta^{(r)}$, we have

$$
\begin{aligned}
& \left|\frac{1}{t-s} \int_{s}^{t} \varphi(u) \vartheta^{(r)}(u) d u-\frac{1}{t-s} \int_{s}^{t} \varphi(u) d u \cdot \frac{1}{t-s} \int_{s}^{t} \vartheta^{(r)}(u) d u\right| \\
& \quad \leq \sqrt{\frac{\Lambda(\varphi(u), \varphi(u))}{2(t-s)}} \cdot \sqrt{\int_{s}^{t}(u-s)(t-u)\left(\vartheta^{(r+1)}(u)\right)^{2} d u}
\end{aligned}
$$


Divide both sides of (3.4) by $(r-3)$ ! and in the obtained expression denote

$$
\Xi_{r}(s, t ; \vartheta)=\frac{1}{(t-s)(r-3) !} \int_{s}^{t} \varphi(u)\left[\vartheta^{(r)}(u)-\frac{\vartheta^{(r-1)}(t)-\vartheta^{(r-1)}(s)}{t-s}\right] d u,
$$

inequality (3.3) is immediate. Now, taking the value of $\frac{1}{(t-s)(r-3) !} \int_{s}^{t} \varphi(u) \vartheta^{(r)}(u) d u$ from (3.5) and substituting in identity (2.1), we have (3.2).

Theorem 3.2 Let $\vartheta:[s, t] \rightarrow \mathbb{R}, \vartheta^{(r)}$ be absolutely continuous for $r \geq 3$, and let $\vartheta^{(r+1)} \geq 0$ on $[s, t]$. Let $\Xi_{r}(s, t ; \vartheta)$ be the same as defined in (3.5). If $\varphi$ is the same as defined in (3.1), then we obtain (3.2) and the remainder $\Xi_{r}(s, t ; \vartheta)$ satisfies

$$
\left|\Xi_{r}(s, t ; \vartheta)\right| \leq \frac{\left\|\varphi^{\prime}(u)\right\|_{\infty}}{(r-3) !}\left[\frac{\vartheta^{(r-1)}(s)+\vartheta^{(r-1)}(t)}{2}-\frac{\vartheta^{(r-2)}(t)-\vartheta^{(r-2)}(s)}{t-s}\right] .
$$

Proof Applying Theorem 1.11 for $\zeta_{1} \rightarrow \varphi$ and $\zeta_{2} \rightarrow \vartheta^{(r)}$, we have

$$
\begin{aligned}
& \left|\frac{1}{t-s} \int_{s}^{t} \varphi(u) \vartheta^{(r)}(u) d u-\frac{1}{t-s} \int_{s}^{t} \varphi(u) d u \cdot \frac{1}{t-s} \int_{s}^{t} \vartheta^{(r)}(u) d u\right| \\
& \quad \leq \frac{1}{2(t-s)}\left\|\varphi^{\prime}(u)\right\|_{\infty} \int_{s}^{t}(u-s)(t-u) \vartheta^{(r+1)}(u) d u .
\end{aligned}
$$

Dividing inequality (3.7) by $(r-3)$ ! and using the expression

$$
\begin{aligned}
& \int_{s}^{t}(u-s)(t-u) \vartheta^{(r+1)}(u) d u \\
& \quad=(t-s)\left(\vartheta^{(r-1)}(s)+\vartheta^{(r-1)}(t)\right)-2\left(\vartheta^{(r-2)}(t)-\vartheta^{(r-2)}(s)\right),
\end{aligned}
$$

we have

$$
\begin{aligned}
\left|\Xi_{r}(s, t ; \vartheta)\right| \leq & \frac{1}{2(t-s)} \cdot \frac{\left\|\varphi^{\prime}(u)\right\|_{\infty}}{(r-3) !}\left[(t-s)\left(\vartheta^{(r-1)}(s)+\vartheta^{(r-1)}(t)\right)\right. \\
& \left.-2\left(\vartheta^{(r-2)}(t)-\vartheta^{(r-2)}(s)\right)\right] .
\end{aligned}
$$

After simplification, inequality (3.8) reduces to (3.6), and by taking the value of $\frac{1}{(t-s)(r-3) !} \int_{s}^{t} \varphi(u) \vartheta^{(r)}(u) d u$ from (3.5) and by inserting into (2.1), we have the representation (3.2).

\section{Ostrowski type inequality related to the obtained identity}

Here we present the Ostrowski type inequality related to the identity given in the second section.

Theorem 4.1 Let all the assumptions of Theorem 2.1 be satisfied, and let $\varphi$ be the same as defined in (3.1). Let $p, q \in[1, \infty]$ such that $\frac{1}{p}+\frac{1}{q}=1$, and let $\left|\vartheta^{(r)}\right|^{p}:[s, t] \rightarrow \mathbb{R}$ be an $\mathbb{R}$ integrable function for some $r \geq 3$. Let $q_{1} a_{1}, \sum_{i=1}^{m} q_{i} a_{i}, Q_{i} a_{i}, Q_{i-1} a_{i} \in[s, t]$ for all $i \in$ 
$\{2, \ldots, m\}$. Then

$$
\begin{aligned}
& \mid \vartheta\left(\sum_{i=1}^{m} q_{i} a_{i}\right)-\vartheta\left(q_{1} a_{1}\right)-\sum_{i=2}^{m}\left[\vartheta\left(Q_{i} a_{i}\right)-\vartheta\left(Q_{i-1} a_{i}\right)\right] \\
& \quad-\frac{1}{t-s} \sum_{n=0}^{r-3} \frac{r-n-2}{n !} \int_{s}^{t}\left[G_{\delta}\left(\sum_{i=1}^{m} q_{i} a_{i}, \tilde{u}\right)-G_{\delta}\left(q_{1} a_{1}, \tilde{u}\right)\right. \\
& \left.\quad-\sum_{i=2}^{m}\left[G_{\delta}\left(Q_{i} a_{i}, \tilde{u}\right)-G_{\delta}\left(Q_{i-1} a_{i}, \tilde{u}\right)\right]\right] \\
& \quad \times\left[(\tilde{u}-t)^{n} \vartheta^{(n+1)}(t)-(\tilde{u}-s)^{n} \vartheta^{(n+1)}(s)\right] d \tilde{u} \mid \\
& \leq\left(\int_{s}^{t}\left|\vartheta^{(r)}(u)\right|^{p} d u\right)^{\frac{1}{p}}\left(\int_{s}^{t}|\dot{\varphi}(u)|^{q} d u\right)^{\frac{1}{q}}
\end{aligned}
$$

where $\dot{\varphi}(u):=\frac{\varphi(u)}{(t-s)(r-3) !}$. The constant $\left(\int_{s}^{t}|\dot{\varphi}(u)|^{q} d u\right)^{\frac{1}{q}}$ in (4.1) is sharp for $1<p \leq \infty$ and best possible for $p=1$.

Proof From identity (2.1), we have

$$
\begin{aligned}
& \mid \vartheta\left(\sum_{i=1}^{m} q_{i} a_{i}\right)-\vartheta\left(q_{1} a_{1}\right)-\sum_{i=2}^{m}\left[\vartheta\left(Q_{i} a_{i}\right)-\vartheta\left(Q_{i-1} a_{i}\right)\right] \\
& \quad-\frac{1}{t-s} \sum_{n=0}^{r-3} \frac{r-n-2}{n !} \int_{s}^{t}\left[G_{\delta}\left(\sum_{i=1}^{m} q_{i} a_{i}, \tilde{u}\right)-G_{\delta}\left(q_{1} a_{1}, \tilde{u}\right)\right. \\
& \left.\quad-\sum_{i=2}^{m}\left[G_{\delta}\left(Q_{i} a_{i}, \tilde{u}\right)-G_{\delta}\left(Q_{i-1} a_{i}, \tilde{u}\right)\right]\right] \\
& \quad \times\left[(\tilde{u}-t)^{n} \vartheta^{(n+1)}(t)-(\tilde{u}-s)^{n} \vartheta^{(n+1)}(s)\right] d \tilde{u} \mid \\
& =\left|\int_{s}^{t} \vartheta^{(r)}(u) \dot{\varphi}(u) d u\right| .
\end{aligned}
$$

On the RHS of (4.2), we apply Hölder's inequality for integrals as follows:

$$
\left|\int_{s}^{t} \vartheta^{(r)}(u) \dot{\varphi}(u) d u\right| \leq\left(\int_{s}^{t}\left|\vartheta^{(r)}(u)\right|^{p} d u\right)^{\frac{1}{p}}\left(\int_{s}^{t}|\dot{\varphi}(u)|^{q} d u\right)^{\frac{1}{q}}
$$

and inequality (4.3) together with (4.2) implies (4.1).

For the proof of the sharpness of the constant $\left(\int_{s}^{t}|\dot{\varphi}(u)|^{q} d u\right)^{\frac{1}{q}}$, we define

$$
\vartheta^{(r)}(u)= \begin{cases}\operatorname{sgn} \dot{\varphi}(u) \cdot|\dot{\varphi}(u)|^{\frac{1}{p-1}}, & 1<p<\infty \\ \operatorname{sgn} \dot{\varphi}(u), & p=\infty\end{cases}
$$

such that the equality in (4.3) holds. 
For $p=1$, we will prove that the following inequality

$$
\left|\int_{s}^{t} \vartheta^{(r)}(u) \dot{\varphi}(u) d u\right| \leq \max _{u \in[s, t]}|\dot{\varphi}(u)| \cdot \int_{s}^{t}\left|\vartheta^{(r)}(u)\right| d u
$$

is the best possible inequality.

Let $|\dot{\varphi}(u)|$ attain its maximum at $u_{0} \in[s, t]$.

Case 1 . When $\dot{\varphi}\left(u_{0}\right)>0$. For small enough $\epsilon$, we define

$$
\vartheta_{\epsilon}(u)= \begin{cases}0, & s \leq u \leq u_{0}, \\ \frac{1}{\epsilon r !}\left(u-u_{0}\right)^{r}, & u_{0} \leq u \leq u_{0}+\epsilon, \\ \frac{1}{(r-1) !}\left(u-u_{0}\right)^{r-1}, & u_{0}+\epsilon \leq u \leq t\end{cases}
$$

Clearly,

$$
\left|\int_{s}^{t} \vartheta_{\epsilon}^{(r)}(u) \dot{\varphi}(u) d u\right|=\frac{1}{\epsilon} \int_{u_{0}}^{u_{0}+\epsilon} \dot{\varphi}(u) d u
$$

and

$$
\int_{s}^{t}\left|\vartheta_{\epsilon}^{(r)}(u)\right| d u=\frac{1}{\epsilon} \int_{u_{0}}^{u_{0}+\epsilon} d u=1
$$

Now, using (4.5) and (4.6) in (4.4) and also using the fact that $|\dot{\varphi}(u)|$ attains its maximum at $u_{0} \in[s, t]$, we have

$$
\frac{1}{\epsilon} \int_{u_{0}}^{u_{0}+\epsilon} \dot{\varphi}(u) d u \leq \dot{\varphi}\left(u_{0}\right) \cdot 1=\dot{\varphi}\left(u_{0}\right) .
$$

As $\lim _{\epsilon \rightarrow 0} \frac{1}{\epsilon} \int_{u_{0}}^{u_{0}+\epsilon} \dot{\varphi}(u) d u=\dot{\varphi}\left(u_{0}\right)$, the statement follows.

Case 2. When $\dot{\varphi}\left(u_{0}\right)<0$, we define

$$
\vartheta_{\epsilon}(u)= \begin{cases}\frac{1}{(r-1) !}\left(u-u_{0}-\epsilon\right)^{r-1}, & s \leq u \leq u_{0} \\ -\frac{1}{\epsilon r !}\left(u-u_{0}-\epsilon\right)^{r}, & u_{0} \leq u \leq u_{0}+\epsilon \\ 0, & u_{0}+\epsilon \leq u \leq t\end{cases}
$$

and the remaining part is the same as above.

Remark 4.2 Remark 2.6 is also valid for Sects. 3 and 4.

\section{Acknowledgements}

The authors would like to thank the referees for their valuable comments and suggestions. The research of the 2 nd author is supported by the Ministry of Education and Science of the Russian Federation (the Agreement number No. 02.a03.21.0008).

\section{Funding}

Not applicable.

Availability of data and materials

Not applicable.

Competing interests

The authors declare that they have no competing interests. 
Authors' contributions

Both the authors contributed to this paper equally and both read and approved the final manuscript.

\section{Author details}

'Department of Mathematics, COMSATS University Islamabad, Lahore Campus, 54000 Lahore, Pakistan. ${ }^{2}$ Rudn University, Miklukho-Maklaya Str. 6, 117198 Moscow, Russia.

\section{Publisher's Note}

Springer Nature remains neutral with regard to jurisdictional claims in published maps and institutional affiliations.

Received: 7 May 2020 Accepted: 6 October 2020 Published online: 21 December 2020

\section{References}

1. Bennett, G.: Lower bounds for matrices. Linear Algebra Appl. 82, 81-98 (1986)

2. Cerone, P., Dragomir, S.S.: Some new Ostrowski-type bounds for the Čebyšev functional and applications. J. Math. Inequal. 8(1), 159-170 (2014)

3. Chebyshev, P.L.: Sur les expressions approximative des integrals par les auters prises entre les mêmes limites. Proc. Math. Soc. Charkov. 2, 93-98 (1882)

4. Fahad, A., Pečarić, J., Qureshi, M.I.: Generalized Steffensen's inequality by Lidstone interpolation and Montogomery's identity. J. Inequal. Appl. 2018, 237, 1-21 (2018)

5. Fink, A.M.: Bounds of the deviation of a function from its averages. Czechoslov. Math. J. 42(117), 289-310 (1992)

6. Hardy, G.H., Littlewood, J.E., Pólya, G.: Inequalities, 2nd edn. Cambridge University Press, Cambridge (1952)

7. Iqbal, A., Khan, M.A., Suleman, M., Chu, Y.: The right Riemann-Liouville fractional Hermite-Hadamard type inequalities derived from Green's function. AIP Adv. 10, 045032 (2020)

8. Khalid, S., Pečarić, Đ., Pečarić, J.: On Shannon and Zipf-Mandelbrot entropies and related results. J. Inequal. Appl. 2019, 99, 1-14 (2019)

9. Khalid, S., Pečarić, Đ., Pečarić, J.: On Zipf-Mandelbrot entropy and 3-convex functions. Adv. Oper. Theory 4, 724-737 (2019)

10. Khalid, S., Pečarić, J., Perić, I.: Refinements and generalizations of majorization, Favard and Berwald-type inequalities via Fink identity. J. Math. Inequal. 12, 267-284 (2018)

11. Khalid, S., Pečarić, J., Praljak, M.: On an inequality of I. Perić. Math. Commun. 19, 221-242 (2014)

12. Khalid, S., Pečarić, J., Vukelić, A.: Refinements of the majorization theorems via Fink identity and related results. J. Class. Anal. 7(2), 129-154 (2015)

13. Khalid, S., Pečarić, J., Vukelić, A.: Refinements of the majorization—type inequalities via Green and Fink identities and related results. Math. Slovaca 68(4), 773-788 (2018)

14. Khan, M.A., Khan, S., Chu, Y.: A new bound for the Jensen gap with applications in information theory. IEEE Access 8 98001-98008 (2020)

15. Khan, M.A., Latif, N., Perić, I., Pečarić, J.: On majorization for matrices. Math. Balk. 27, 3-19 (2013)

16. Khan, M.A., Mohammad, N., Nwaeze, E.R., Chu, Y.: Quantum Hermite-Hadamard inequality by means of a Green function. Adv. Differ. Equ. 2020, 99, 1-20 (2020)

17. Khan, S., Khan, M.A., Butt, S.I., Chu, Y.: A new bound for the Jensen gap pertaining twice differentiable functions with applications. Adv. Differ. Equ. 2020, 333, 1-11 (2020)

18. Khan, S., Khan, M.A., Chu, Y.: New converses of Jensen inequality via Green functions with applications. Rev. R. Acad. Cienc. Exactas Fís. Nat., Ser. A Mat. 114, 114 (2020)

19. Khan, S., Khan, M.A., Chu, Y.: Converses of the Jensen inequality derived from the Green functions with applications in information theory. Math. Methods Appl. Sci. 43, 2577-2587 (2020)

20. Khurshid, Y., Khan, M.A., Chu, Y.: Ostrowski type inequalities involving conformable integrals via preinvex functions. AIP Adv. 10, 055204 (2020)

21. Khurshid, Y., Khan, M.A., Chu, Y.: Conformable fractional integral inequalities for GG- and GA-convex functions. AIMS Math. 5(5), 5012-5030 (2020)

22. Pečarić, Đ., Pečarić, J.: Inequalities and Zipf-Mandelbrot Law: Selected Topics in Information Theory. Monographs in Inequalities, vol. 15. Element d.o.o., Croatia (2019)

23. Pečarić, J., Proschan, F., Tong, Y.L.: Convex Functions, Partial Orderings, and Statistical Applications. Academic Press, San Diego (1992 